\title{
The Method of Position-Tagged Spectrometry
}

\author{
J. J. Friel,* G. H. Nelson, * R. E. Tomenchok, * T. Wallmark, * and R. B. Mott** \\ *Princeton Gamma-Tech, C/N 863, Princeton, NJ 08542 \\ **Acuity Engineering
}

Ever since the early years of scanning microscopy, x-ray mapping has been an important way to determine the spatial distributions of elements within a specimen [1]. The first example of expanding into hyperspectral space was accomplished by Legge and Hammond on a PIXE [2]. They devised a method of collecting full x-ray spectral data in sync with the scanning beam. Their system generated a stream of position-tagged x-ray energies that were spooled to magnetic tape in arrivaltime order and sorted later into a spectrum-image.

The method of position-tagged spectrometry (PTS) as defined by Mott [3,4,5] was invented independently and employs the same principle but performs the functions in real time. The method consists of controlling the electron beam, acquiring x-ray data, and coordinating the timing of all events, as shown schematically in Figure 1. The dedicated x-ray electronics control the parameters of the EDS detector and pulse processing, and data are sent over a parallel interface to the imaging electronics. The imaging board controls the timing, beam driving, and image acquisition, and it stitches together the various types of data to be sent to the host PC over Ethernet.

The host computer sets the collection parameters, creates a dedicated high-priority data processing thread, and creates a sink for all the incoming high-speed data. When the collection process begins, the computer waits and listens for data; as the packets arrive, they are separated into their components and stored in memory. The software also uses separate forms to set up the displays for images, maps, and spectra, all automatically scaled and displaying data in real time. The 3-D PTS data structure is maintained in virtual memory with variable plane depth for efficiency.

Because plane depth is variable, the size of the file depends on the amount of data. A spectral channel can contain any number of counts from 0 to $2^{32}$. The number of channels can be as few as 256 or as many as 4096 . The image resolution does not have to be the same as the map resolution. It can be as low as $64 \times 64$ or as high as 4096x4096, with each cell in the structure capable of holding up to 32 bits. The PTS method would permit 4096-channel spectra in a 4096x4096 image with each pixel containing 32 bits of data-even though the physics x-ray generation and limitations of hardware would preclude so enormous a data structure. Typical file sizes vary from 2 MB to $40 \mathrm{MB}$ depending mostly on image resolution.

While PTS data collection is running, elemental maps can be added or deleted on the fly, and live spectra can be displayed from different regions simultaneously. Even some of what are usually considered post-processing operations can be done live. For example, AutoPhasing can be a time consuming step to sort the complete spectrum image into phases based on x-ray intensity. However, AutoPhase by recursive pixel allocation associated with PTS can be run while the spectrum image is still being collected and the phases are computed up to that time [6]. Moreover, it can be run repeatedly on the live data as x-ray counting statistics improve. 
In addition to AutoPhase, other post-processing operations performed on the spectrum image after collection include: spectrum from a region, maps for any element, and user-constrained phasing.

Spectra can be summed from any set of pixels. These regions can be defined with annotation objects, such as boxes circles, or polygons by AutoPhase, or any image processing operation. For example, one could display a spectrum of all the grain boundary phases, a spectrum along an interface, or even a radial concentration gradient across particles or fibers [7].

Maps for any element extracted from the spectrum image are a straightforward consequence of a spectrum at every pixel. User-constrained phasing is an operation in which one puts tags on specific areas of the electron image, and the computer defines a number of phases equal to the number of tags, and forces all pixels into a best-fit phase. If the operator knows the phase assemblage or mineral assemblage, then spurious phases and boundary phases are eliminated by this method.

In summary, PTS is a method of materials characterization that combines the operations of image processing and analysis with those of x-ray microanalysis. The techniques are complementary, and allow the extraction of size, shape, and compositional data, much of it in real time.

\section{References}

[1] P. Duncumb, X-ray Microscopy and Microradiography (1957) 617.

[2] G. J. F. Legge and I. Hammond, J. Microsc. 117 (1979) 201.

[3] R. B. Mott, et al., Microsc. Microanal. (1995) 592.

[4] R. B. Mott and J. J. Friel, MRS Spring Mtg. (1996) 435.

[5] R. B. Mott and J. J. Friel, J. Microsc. 193 (1999) 2.

[6] J. J. Friel, and R. Batcheler, Microsc. Microanal. (2002) 350.

[7] J. J. Friel, and V. A. Greenhut, J. Am. Cer. S. (1997) 3205.

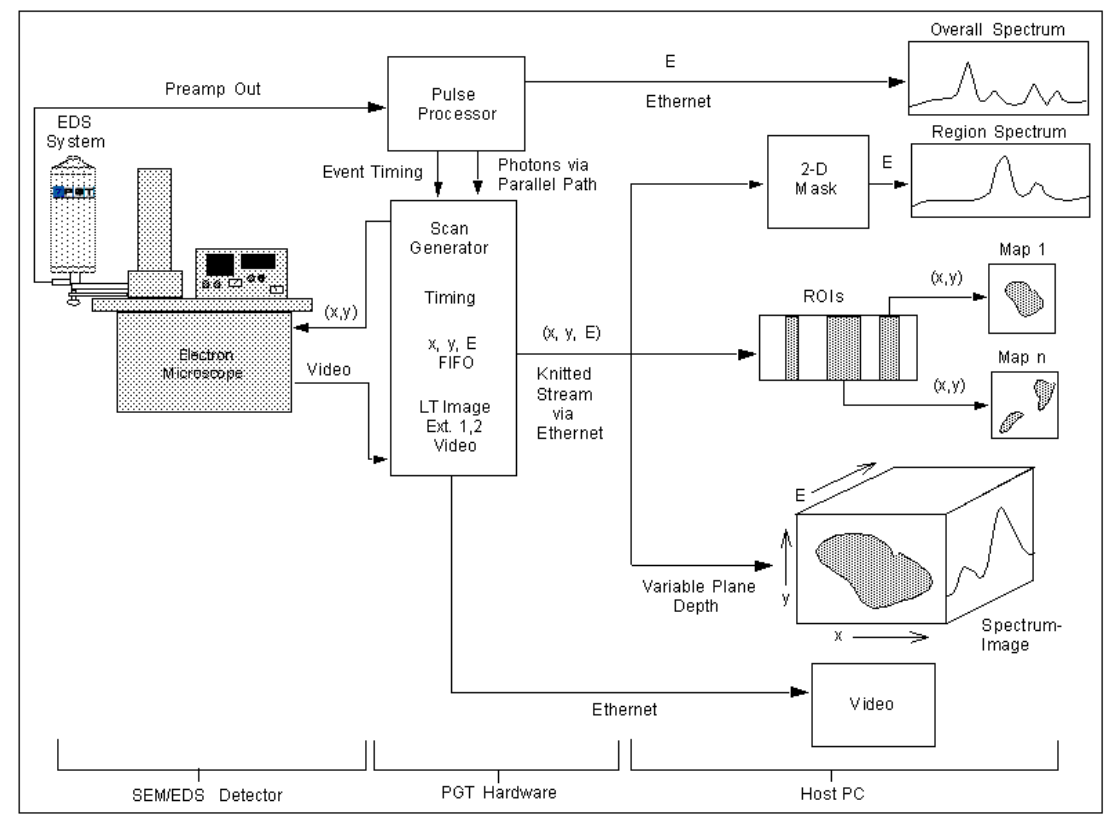

FIG. 1 Schematic diagram showing the interaction of modules and data paths in PTS. 